\title{
Global change in flood and drought intensities under climate change in the $21^{\text {st }}$ century
}

\author{
Behzad Asadieh ${ }^{1,}$ *, Nir Y. Krakauer ${ }^{1}$ \\ 1 Civil Engineering Department and NOAA-CREST, The City College of New York, the City University of \\ New York, New York, USA; basadie00@citymail.cuny.edu; nkrakauer@ccny.cuny.edu \\ * Correspondence Behzad Asadieh: basadie00@citymail.cuny.edu
}

\section{Supplementary Information}

The average value of change in flood and drought indicators for each GCM-GHM combination, under RCP8.5 and RCP2.6 warming scenarios, are presented individually in the Tables S2 and S3, respectively. It should be noted that the results presented in the Tables S2 and S3, if averaged over the models for each quadrant, may result in different values from the multi-model average values reported in the main text. For the values reported in the main text, the results are first averaged over the models for each grid cell to create a multi-model global matrix. The grid-cells are then averaged over each quadrant, weighted by grid cell area. Assignment of each grid cell to the specified quadrants is based on the multi-model averages. For any single model datasets, grid cells (especially the ones with small multimodel mean projected changes) may fall in different quadrants, due to the disagreement among the GCMs and GHMs on projection of changes.

Some ISI-MIP hydrological impact models do not simulate streamflow for some grid cells for which other models do. This is due to the differences in the utilized global river network. Hence, a minority of grid cells do not have data from all hydrological models. However, grid cells with less than 20 (out of 25) available models are excluded from the calculations.

The results presented in the main text are based on the $95^{\text {th }}$ and $5^{\text {th }}$ percentiles of streamflow. Maps of changes in the median of streamflow are also presented in this supplement, along with the maps of changes in $95^{\text {th }}$ and $5^{\text {th }}$ percentiles.

The two-sample t-test (Snedecor and Cochran, 1989) is used in this study to quantify the statistical significance level of difference between the means of the $20 \mathrm{C}$ and $21 \mathrm{C}$ streamflow time series:

$$
T=\frac{\bar{Q}_{21 C}-\bar{Q}_{20 C}}{\sqrt{\frac{\sigma_{21 C}}{N_{21 C}}+\frac{\sigma_{20 C}}{N_{20 C}}}}
$$

where $\bar{Q}_{21 C}$ and $\bar{Q}_{20 C}$ are the mean (high or low) streamflow, $\sigma_{21 C}$ and $\sigma_{21 C}$ are the streamflow time series variances, and $N_{21 C}$ and $N_{20 C}$ are the streamflow time series sample sizes (equal to 30 -years in this study), in $21 \mathrm{C}$ and $20 \mathrm{C}$, respectively. The means are significantly different if the absolute value of $T$ is larger than the critical value of the t-distribution with $v$ degrees of freedom, with $v$ in an equal variance assumption being equal to $N_{21 C}+N_{20 C}-2$. The critical value is defined based on the confidence level of significance and sample size, and at $95 \%$ level for a sample size of 30 it is equal to 2.042 . The percentage of land area with statistically significant change (at $95 \%$ confidence level) is reported in this study. 


\section{Supplementary References:}

Schewe, J., Heinke, J., Gerten, D., Haddeland, I., Arnell, N. W., Clark, D. B., Dankers, R., Eisner, S., Fekete, B. M., Colón-González, F. J., Gosling, S. N., Kim, H., Liu, X., Masaki, Y., Portmann, F. T., Satoh, Y., Stacke, T., Tang, Q., Wada, Y., Wisser, D., Albrecht, T., Frieler, K., Piontek, F., Warszawski, L. and Kabat, P.: Multimodel assessment of water scarcity under climate change, Proc. Natl. Acad. Sci., 111(9), 3245-3250, doi:10.1073/pnas.1222460110, 2013.

Snedecor, G. W. and Cochran, W. G.: Statistical methods, 8thEdn, Iowa State University Press, Iowa., 1989.

Table S1. Main characteristics of the global hydrological models used in this study, obtained from ref. 1 (Schewe et al., 2013). LW: downwelling long-wave radiation; LWn: net long-wave radiation; P: precipitation rate (rain and snow calculated in the model); Q: air specific humidity; R: rainfall rate; RH: relative humidity; S: snowfall rate; SP: surface pressure; SW: downwelling shortwave radiation; $\mathrm{T}$ : air temperature; $\operatorname{Tmax}(\min )$ : daily maximum (minimum) air temperature; W: wind speed.

\begin{tabular}{|l|l|l|l|l|l|l|l|l|}
\hline $\begin{array}{l}\text { Model } \\
\text { name }\end{array}$ & $\begin{array}{l}\text { Time } \\
\text { step }\end{array}$ & $\begin{array}{l}\text { Meteorological } \\
\text { forcing }\end{array}$ & $\begin{array}{l}\text { Energy } \\
\text { balance }\end{array}$ & $\begin{array}{l}\text { Evaporation } \\
\text { scheme }\end{array}$ & $\begin{array}{l}\text { Runoff } \\
\text { scheme }\end{array}$ & $\begin{array}{l}\text { Snow } \\
\text { scheme }\end{array}$ & $\begin{array}{l}\text { Vegetation } \\
\text { dynamics }\end{array}$ & $\begin{array}{l}\text { CO2 } \\
\text { effect }\end{array}$ \\
\hline DBH & 1hour & $\begin{array}{l}\text { P, T, W, Q, LW, } \\
\text { SW, SP }\end{array}$ & Yes & $\begin{array}{l}\text { Energy } \\
\text { balance }\end{array}$ & $\begin{array}{l}\text { Infiltration } \\
\text { excess }\end{array}$ & $\begin{array}{l}\text { Energy } \\
\text { balance }\end{array}$ & No & Constant \\
\hline LPJmL & Daily & P, T, LWn, SW & Yes & $\begin{array}{l}\text { Priestley- } \\
\text { Taylor }\end{array}$ & $\begin{array}{l}\text { Saturation } \\
\text { excess }\end{array}$ & $\begin{array}{l}\text { Degree- } \\
\text { day }\end{array}$ & Yes & Varying \\
\hline Mac-PDM & Daily & $\begin{array}{l}\text { P, T, W,Q, LWn, } \\
\text { SW, SP }\end{array}$ & No & $\begin{array}{l}\text { Penman- } \\
\text { Monteith }\end{array}$ & $\begin{array}{l}\text { Saturation } \\
\text { excess, } \\
\text { non-linear }\end{array}$ & $\begin{array}{l}\text { Degree- } \\
\text { day }\end{array}$ & No & No \\
\hline WBM & Daily & P, T & No & Hamon & $\begin{array}{l}\text { Excess Beta } \\
\text { Function }\end{array}$ & $\begin{array}{l}\text { Degree- } \\
\text { day }\end{array}$ & No \\
\hline Daily & P, T & No & Hamon & $\begin{array}{l}\text { Saturation } \\
\text { Excess }\end{array}$ & $\begin{array}{l}\text { Empirical } \\
\text { temp and } \\
\text { Precip- } \\
\text { based } \\
\text { formula }\end{array}$ & No & No \\
\hline
\end{tabular}


Table S2. Change in flood and drought indicators, averaged for each quadrant (normalized \%). Results presented for each model under RCP8.5 scenario. The results can also be reverted to the relative change in percentage for more distinct comparison (Figure S1).

\begin{tabular}{|c|c|c|c|c|c|c|c|c|c|}
\hline \multicolumn{2}{|c|}{ RCP 8.5} & \multicolumn{2}{|c|}{$\begin{array}{l}\text { Quad. 1. } \\
\text { flood and drought } \\
\text { increased }\end{array}$} & \multicolumn{2}{|c|}{$\begin{array}{c}\text { Quad. 2. } \\
\text { flood increased, } \\
\text { drought decreased }\end{array}$} & \multicolumn{2}{|c|}{$\begin{array}{l}\text { Quad. } 3 . \\
\text { drought increased, } \\
\text { flood decreased }\end{array}$} & \multicolumn{2}{|c|}{$\begin{array}{c}\text { Quad. } 4 . \\
\text { flood and drought } \\
\text { decreased }\end{array}$} \\
\hline GCM & GHM & $\begin{array}{l}\text { Change } \\
\text { in flood }\end{array}$ & $\begin{array}{c}\text { Change } \\
\text { in } \\
\text { drought }\end{array}$ & $\begin{array}{l}\text { Change } \\
\text { in flood }\end{array}$ & $\begin{array}{l}\text { Change } \\
\text { in } \\
\text { drought }\end{array}$ & $\begin{array}{l}\text { Change } \\
\text { in flood }\end{array}$ & $\begin{array}{c}\text { Change } \\
\text { in } \\
\text { drought }\end{array}$ & $\begin{array}{l}\text { Change } \\
\text { in flood }\end{array}$ & $\begin{array}{c}\text { Change } \\
\text { in } \\
\text { drought }\end{array}$ \\
\hline \multirow{5}{*}{$\begin{array}{c}\text { GFDL-ESM2 } \\
\mathrm{m}\end{array}$} & WBM & $7.2 \%$ & $17.8 \%$ & $19.6 \%$ & $-29.1 \%$ & $-28.5 \%$ & $45.1 \%$ & $-10.5 \%$ & $-17.1 \%$ \\
\hline & MacPDM & $5.6 \%$ & $10.8 \%$ & $15.2 \%$ & $-20.6 \%$ & $-16.7 \%$ & $29.4 \%$ & $-6.6 \%$ & $-18.9 \%$ \\
\hline & $\begin{array}{c}\text { PCR- } \\
\text { GLOBWB }\end{array}$ & $7.6 \%$ & $17.5 \%$ & $15.0 \%$ & $-18.6 \%$ & $-23.5 \%$ & $39.3 \%$ & $-12.1 \%$ & $-17.2 \%$ \\
\hline & $\mathrm{DBH}$ & $7.9 \%$ & $7.4 \%$ & $12.9 \%$ & $-21.4 \%$ & $-16.1 \%$ & $16.9 \%$ & $-10.1 \%$ & $-31.4 \%$ \\
\hline & LPJmL & $7.9 \%$ & $19.1 \%$ & $12.9 \%$ & $-23.1 \%$ & $-16.1 \%$ & $40.5 \%$ & $-8.8 \%$ & $-25.1 \%$ \\
\hline \multirow{5}{*}{ HadGEM2-ES } & WBM & $9.1 \%$ & $28.7 \%$ & $30.6 \%$ & $-47.7 \%$ & $-27.1 \%$ & $49.6 \%$ & $-9.5 \%$ & $-24.6 \%$ \\
\hline & MacPDM & $5.1 \%$ & $7.7 \%$ & $17.2 \%$ & $-22.4 \%$ & $-14.8 \%$ & $27.0 \%$ & $-8.0 \%$ & $-19.2 \%$ \\
\hline & $\begin{array}{c}\text { PCR- } \\
\text { GLOBWB }\end{array}$ & $10.2 \%$ & $25.0 \%$ & $22.6 \%$ & $-29.2 \%$ & $-26.8 \%$ & $47.4 \%$ & $-14.2 \%$ & $-21.8 \%$ \\
\hline & DBH & $10.3 \%$ & $8.7 \%$ & $16.6 \%$ & $-30.1 \%$ & $-15.3 \%$ & $20.3 \%$ & $-9.4 \%$ & $-41.5 \%$ \\
\hline & LPJmL & $11.0 \%$ & $22.0 \%$ & $17.6 \%$ & $-26.7 \%$ & $-14.1 \%$ & $41.2 \%$ & $-9.6 \%$ & $-28.8 \%$ \\
\hline \multirow{5}{*}{$\begin{array}{c}\text { IPSL-CM5A- } \\
\text { LR }\end{array}$} & WBM & $9.5 \%$ & $22.6 \%$ & $27.8 \%$ & $-42.3 \%$ & $-35.7 \%$ & $56.3 \%$ & $-11.4 \%$ & $-20.1 \%$ \\
\hline & MacPDM & $5.8 \%$ & $8.7 \%$ & $17.9 \%$ & $-21.6 \%$ & $-19.0 \%$ & $30.2 \%$ & $-6.6 \%$ & $-14.3 \%$ \\
\hline & $\begin{array}{c}\text { PCR- } \\
\text { GLOBWB }\end{array}$ & $10.8 \%$ & $24.1 \%$ & $24.1 \%$ & $-25.4 \%$ & $-28.9 \%$ & $48.1 \%$ & $-15.5 \%$ & $-22.0 \%$ \\
\hline & DBH & $11.2 \%$ & $8.1 \%$ & $20.0 \%$ & $-28.1 \%$ & $-19.1 \%$ & $20.0 \%$ & $-12.6 \%$ & $-44.4 \%$ \\
\hline & LPJmL & $12.8 \%$ & $21.1 \%$ & $18.8 \%$ & $-27.8 \%$ & $-18.7 \%$ & $39.4 \%$ & $-11.0 \%$ & $-27.9 \%$ \\
\hline \multirow{5}{*}{ MIROC-ESM } & WBM & $9.2 \%$ & $21.5 \%$ & $26.7 \%$ & $-42.3 \%$ & $-34.0 \%$ & $52.7 \%$ & $-8.9 \%$ & $-20.4 \%$ \\
\hline & MacPDM & $4.1 \%$ & $6.1 \%$ & $11.6 \%$ & $-13.4 \%$ & $-15.0 \%$ & $25.9 \%$ & $-3.8 \%$ & $-8.7 \%$ \\
\hline & $\begin{array}{c}\text { PCR- } \\
\text { GLOBWB }\end{array}$ & $10.3 \%$ & $26.1 \%$ & $22.3 \%$ & $-27.0 \%$ & $-27.2 \%$ & $44.3 \%$ & $-13.7 \%$ & $-19.4 \%$ \\
\hline & $\mathrm{DBH}$ & $10.1 \%$ & $8.9 \%$ & $17.3 \%$ & $-32.2 \%$ & $-18.0 \%$ & $18.2 \%$ & $-10.9 \%$ & $-39.7 \%$ \\
\hline & LPJmL & $11.6 \%$ & $20.0 \%$ & $16.0 \%$ & $-29.9 \%$ & $-16.4 \%$ & $38.9 \%$ & $-10.4 \%$ & $-29.9 \%$ \\
\hline \multirow{5}{*}{ NorESM1-M } & WBM & $8.1 \%$ & $21.5 \%$ & $18.9 \%$ & $-34.5 \%$ & $-25.5 \%$ & $42.9 \%$ & $-8.2 \%$ & $-20.4 \%$ \\
\hline & MacPDM & $3.8 \%$ & $6.3 \%$ & $11.1 \%$ & $-15.1 \%$ & $-12.7 \%$ & $21.8 \%$ & $-7.7 \%$ & $-11.1 \%$ \\
\hline & $\begin{array}{c}\text { PCR- } \\
\text { GLOBWB }\end{array}$ & $7.2 \%$ & $21.0 \%$ & $15.2 \%$ & $-21.8 \%$ & $-21.9 \%$ & $37.7 \%$ & $-10.5 \%$ & $-19.1 \%$ \\
\hline & DBH & $7.4 \%$ & $5.8 \%$ & $12.1 \%$ & $-20.2 \%$ & $-14.0 \%$ & $12.2 \%$ & $-7.2 \%$ & $-35.6 \%$ \\
\hline & LPJmL & $7.7 \%$ & $20.1 \%$ & $12.0 \%$ & $-23.7 \%$ & $-13.3 \%$ & $30.9 \%$ & $-10.3 \%$ & $-24.4 \%$ \\
\hline
\end{tabular}


Table S3. Change in flood and drought indicators, averaged for each quadrant (normalized \%). Results presented for each model under RCP2.6 scenario. The results can also be reverted to the relative change in percentage for more distinct comparison (Figure S1).

\begin{tabular}{|c|c|c|c|c|c|c|c|c|c|}
\hline \multicolumn{2}{|c|}{ RCP 2.6} & \multicolumn{2}{|c|}{$\begin{array}{l}\text { Quad. 1. } \\
\text { flood and drought } \\
\text { increased }\end{array}$} & \multicolumn{2}{|c|}{$\begin{array}{c}\text { Quad. 2. } \\
\text { flood increased, } \\
\text { drought decreased }\end{array}$} & \multicolumn{2}{|c|}{$\begin{array}{l}\text { Quad. } 3 . \\
\text { drought increased, } \\
\text { flood decreased }\end{array}$} & \multicolumn{2}{|c|}{$\begin{array}{c}\text { Quad. } 4 . \\
\text { flood and drought } \\
\text { decreased }\end{array}$} \\
\hline GCM & GHM & $\begin{array}{l}\text { Change } \\
\text { in flood }\end{array}$ & $\begin{array}{c}\text { Change } \\
\text { in } \\
\text { drought }\end{array}$ & $\begin{array}{l}\text { Change } \\
\text { in flood }\end{array}$ & $\begin{array}{l}\text { Change } \\
\text { in } \\
\text { drought }\end{array}$ & $\begin{array}{l}\text { Change } \\
\text { in flood }\end{array}$ & $\begin{array}{c}\text { Change } \\
\text { in } \\
\text { drought }\end{array}$ & $\begin{array}{l}\text { Change } \\
\text { in flood }\end{array}$ & $\begin{array}{c}\text { Change } \\
\text { in } \\
\text { drought }\end{array}$ \\
\hline \multirow{5}{*}{$\begin{array}{c}\text { GFDL-ESM2 } \\
\mathrm{m}\end{array}$} & WBM & $6.7 \%$ & $14.3 \%$ & $15.3 \%$ & $-22.4 \%$ & $-15.3 \%$ & $27.0 \%$ & $-6.3 \%$ & $-13.4 \%$ \\
\hline & MacPDM & $3.5 \%$ & $10.8 \%$ & $9.4 \%$ & $-13.7 \%$ & $-10.4 \%$ & $17.1 \%$ & $-3.2 \%$ & $-8.1 \%$ \\
\hline & $\begin{array}{c}\text { PCR- } \\
\text { GLOBWB }\end{array}$ & $5.5 \%$ & $12.0 \%$ & $11.0 \%$ & $-17.3 \%$ & $-12.3 \%$ & $22.8 \%$ & $-6.5 \%$ & $-12.5 \%$ \\
\hline & $\mathrm{DBH}$ & $4.6 \%$ & $3.7 \%$ & $8.6 \%$ & $-11.6 \%$ & $-7.7 \%$ & $6.8 \%$ & $-4.4 \%$ & $-12.8 \%$ \\
\hline & LPJmL & $4.2 \%$ & $16.2 \%$ & $7.8 \%$ & $-15.8 \%$ & $-6.5 \%$ & $23.7 \%$ & $-4.5 \%$ & $-17.8 \%$ \\
\hline \multirow{5}{*}{ HadGEM2-ES } & WBM & $6.9 \%$ & $14.9 \%$ & $18.4 \%$ & $-29.7 \%$ & $-15.1 \%$ & $27.9 \%$ & $-6.2 \%$ & $-14.1 \%$ \\
\hline & MacPDM & $3.1 \%$ & $5.2 \%$ & $9.7 \%$ & $-13.6 \%$ & $-6.8 \%$ & $12.5 \%$ & $-3.3 \%$ & $-7.2 \%$ \\
\hline & $\begin{array}{c}\text { PCR- } \\
\text { GLOBWB }\end{array}$ & $7.2 \%$ & $15.4 \%$ & $14.1 \%$ & $-18.1 \%$ & $-14.5 \%$ & $27.1 \%$ & $-8.0 \%$ & $-14.3 \%$ \\
\hline & DBH & $5.9 \%$ & $4.8 \%$ & $10.5 \%$ & $-14.2 \%$ & $-6.8 \%$ & $8.2 \%$ & $-4.4 \%$ & $-16.3 \%$ \\
\hline & LPJmL & $7.3 \%$ & $16.5 \%$ & $10.6 \%$ & $-18.3 \%$ & $-6.1 \%$ & $23.3 \%$ & $-4.9 \%$ & $-18.2 \%$ \\
\hline \multirow{5}{*}{$\begin{array}{c}\text { IPSL-CM5A- } \\
\text { LR }\end{array}$} & WBM & $5.5 \%$ & $12.4 \%$ & $14.1 \%$ & $-23.5 \%$ & $-17.0 \%$ & $27.8 \%$ & $-8.8 \%$ & $-13.8 \%$ \\
\hline & MacPDM & $3.3 \%$ & $5.4 \%$ & $8.2 \%$ & $-10.3 \%$ & $-8.8 \%$ & $14.0 \%$ & $-2.6 \%$ & $-7.1 \%$ \\
\hline & $\begin{array}{c}\text { PCR- } \\
\text { GLOBWB }\end{array}$ & $5.5 \%$ & $13.1 \%$ & $10.8 \%$ & $-14.4 \%$ & $-16.1 \%$ & $27.4 \%$ & $-9.1 \%$ & $-14.9 \%$ \\
\hline & DBH & $5.5 \%$ & $4.6 \%$ & $9.8 \%$ & $-14.2 \%$ & $-9.2 \%$ & $9.5 \%$ & $-4.5 \%$ & $-19.0 \%$ \\
\hline & LPJmL & $6.0 \%$ & $16.5 \%$ & $8.4 \%$ & $-18.0 \%$ & $-7.8 \%$ & $25.1 \%$ & $-5.5 \%$ & $-16.2 \%$ \\
\hline \multirow{5}{*}{ MIROC-ESM } & WBM & $5.6 \%$ & $15.6 \%$ & $17.5 \%$ & $-26.3 \%$ & $-20.8 \%$ & $32.9 \%$ & $-6.3 \%$ & $-13.5 \%$ \\
\hline & MacPDM & $3.1 \%$ & $4.0 \%$ & $7.3 \%$ & $-10.6 \%$ & $-7.4 \%$ & $12.6 \%$ & $-1.9 \%$ & $-4.7 \%$ \\
\hline & $\begin{array}{c}\text { PCR- } \\
\text { GLOBWB }\end{array}$ & $6.3 \%$ & $15.9 \%$ & $14.6 \%$ & $-19.6 \%$ & $-17.6 \%$ & $28.9 \%$ & $-7.2 \%$ & $-13.5 \%$ \\
\hline & $\mathrm{DBH}$ & $5.7 \%$ & $5.9 \%$ & $11.4 \%$ & $-15.7 \%$ & $-10.2 \%$ & $9.3 \%$ & $-7.4 \%$ & $-20.1 \%$ \\
\hline & LPJmL & $6.3 \%$ & $17.2 \%$ & $9.1 \%$ & $-19.0 \%$ & $-9.4 \%$ & $27.2 \%$ & $-6.7 \%$ & $-18.4 \%$ \\
\hline \multirow{5}{*}{ NorESM1-M } & WBM & $4.5 \%$ & $11.6 \%$ & $12.9 \%$ & $-20.5 \%$ & $-14.7 \%$ & $23.5 \%$ & $-4.9 \%$ & $-11.2 \%$ \\
\hline & MacPDM & $2.3 \%$ & $4.8 \%$ & $6.4 \%$ & $-8.2 \%$ & $-6.3 \%$ & $11.8 \%$ & $-2.4 \%$ & $-5.4 \%$ \\
\hline & $\begin{array}{c}\text { PCR- } \\
\text { GLOBWB }\end{array}$ & $5.1 \%$ & $12.4 \%$ & $8.7 \%$ & $-12.9 \%$ & $-13.3 \%$ & $23.9 \%$ & $-5.4 \%$ & $-11.2 \%$ \\
\hline & DBH & $5.0 \%$ & $3.6 \%$ & $6.9 \%$ & $-11.4 \%$ & $-8.2 \%$ & $6.8 \%$ & $-3.6 \%$ & $-12.2 \%$ \\
\hline & LPJmL & $4.1 \%$ & $15.5 \%$ & $6.2 \%$ & $-14.5 \%$ & $-5.6 \%$ & $21.1 \%$ & $-4.8 \%$ & $-14.9 \%$ \\
\hline
\end{tabular}


Global increased flood and drought risks under climate change in the 21st century

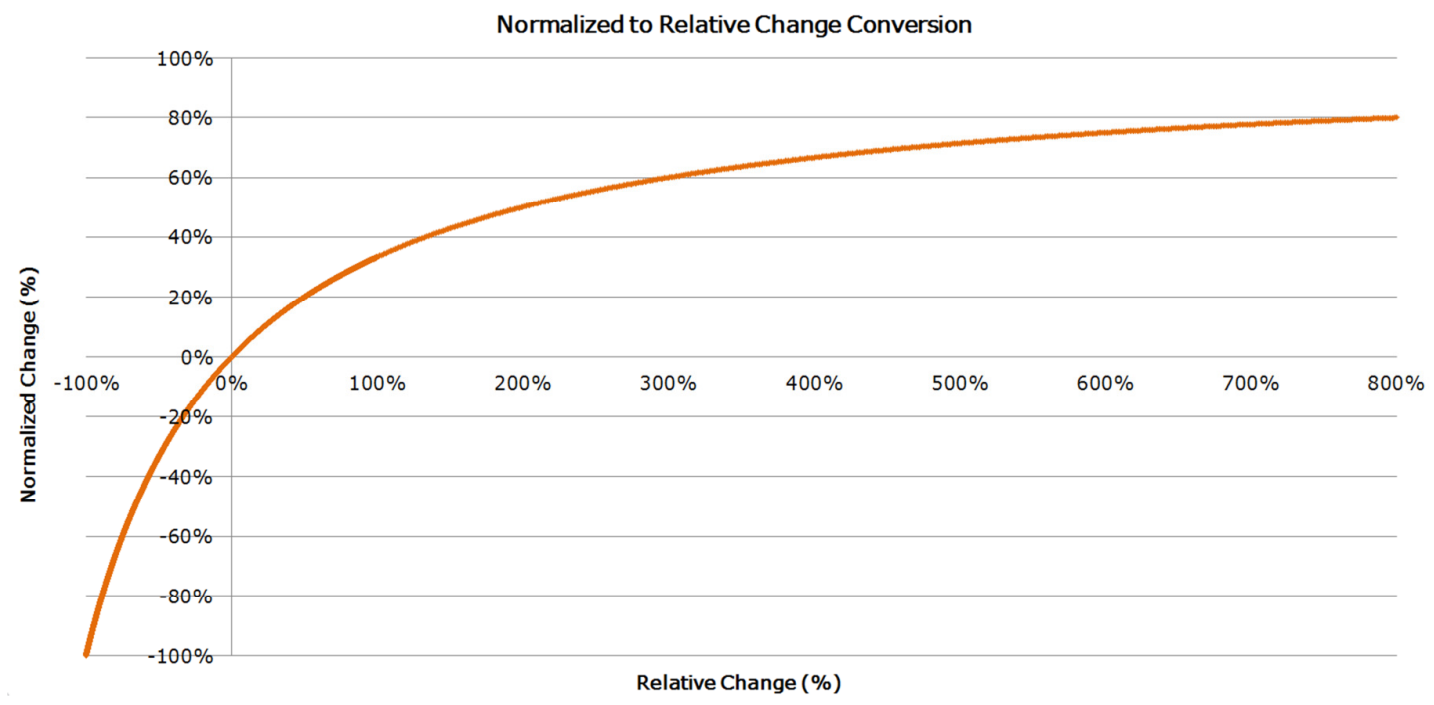

Figure S1. Normalized change (\%) versus relative change (\%) curve. 


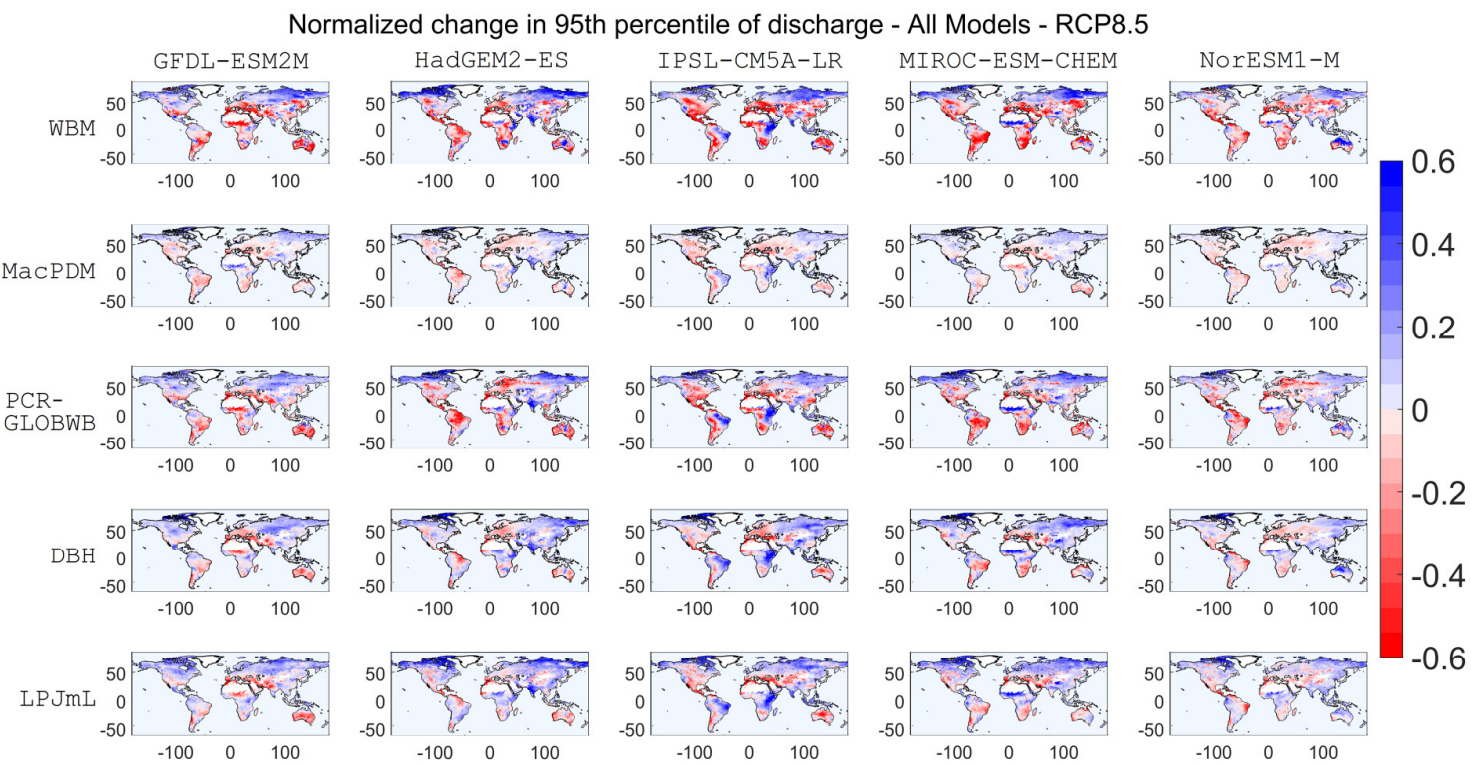

Figure S2. Normalized change in P95 for each of GHM/GCM combinations under RCP8.5 scenario (flood indicator).

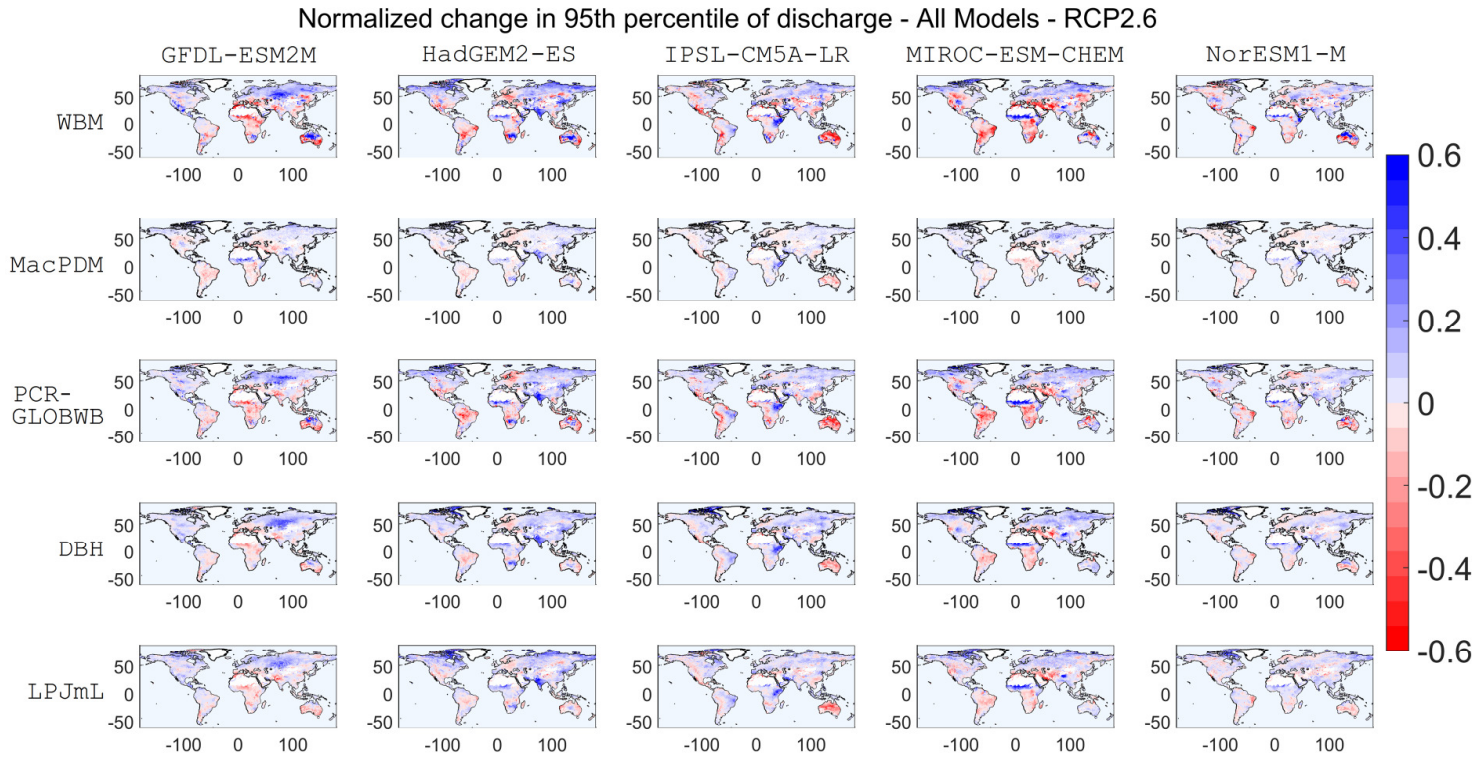

Figure S3. Normalized change in P95 for each of GHM/GCM combinations under RCP2.6 scenario (flood indicator). 


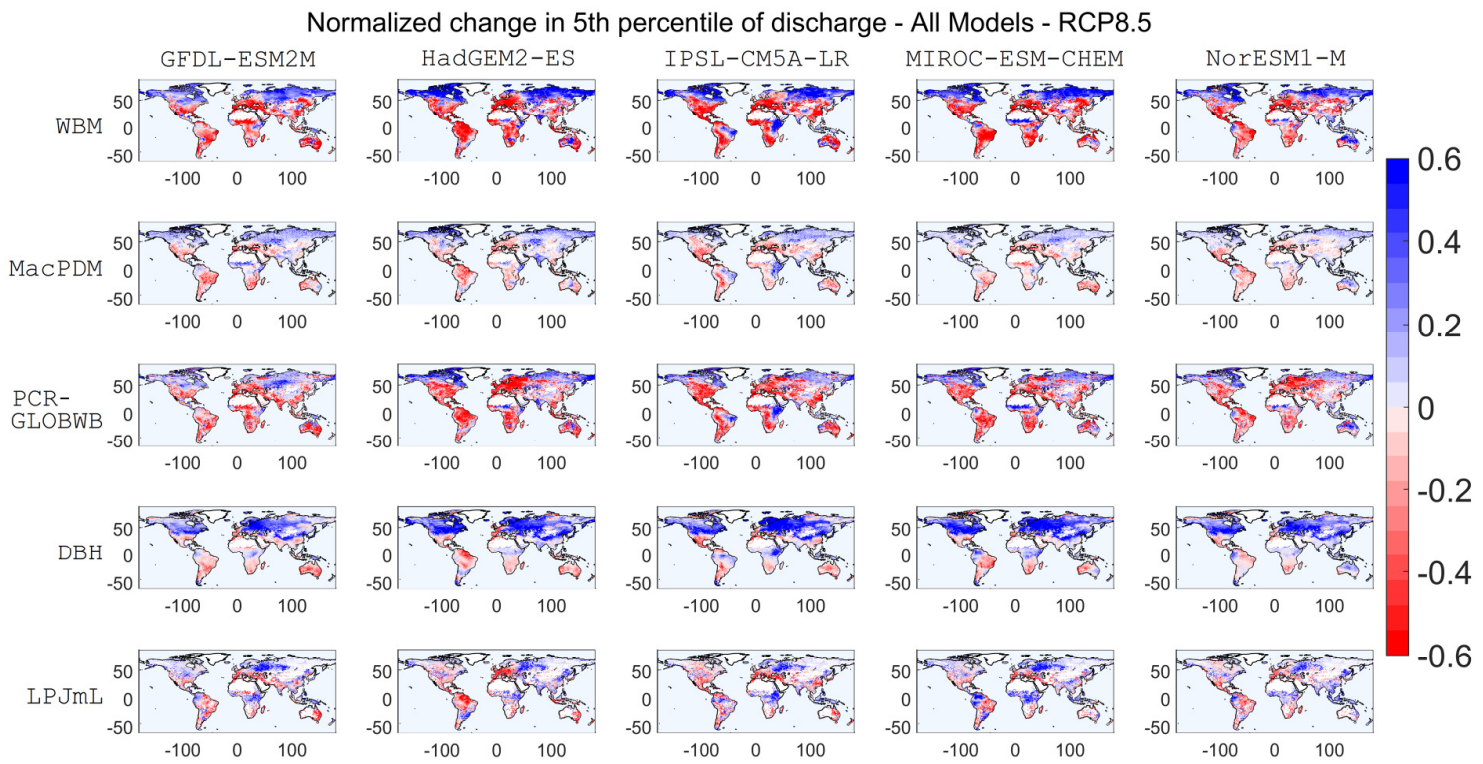

Figure S4. Normalized change in P5 for each of GHM/GCM combinations under RCP8.5 scenario. It should be noted that the drought indicator is defined as the change in the P5 multiplied by -1 .

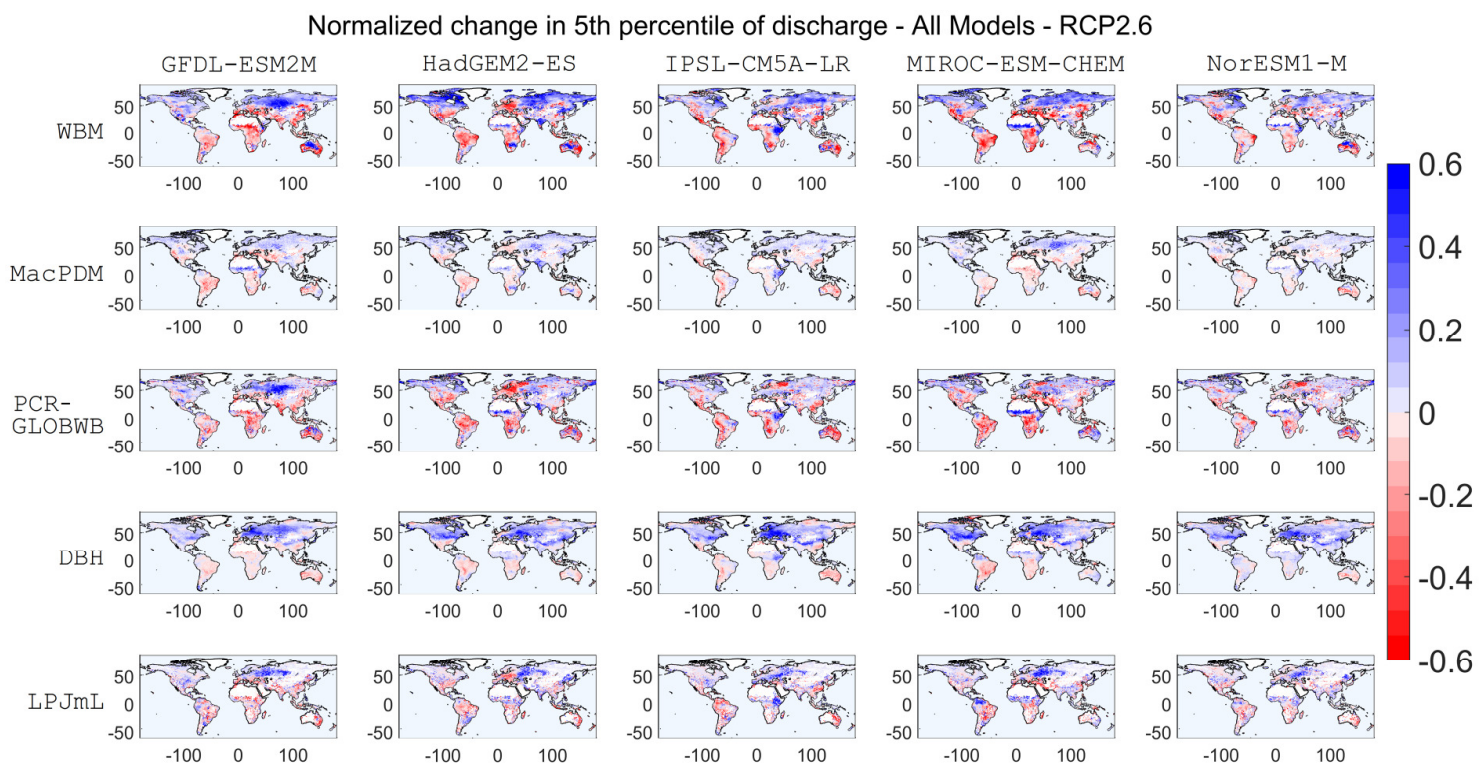

Figure S5. Normalized change in P5 for each of GHM/GCM combinations under RCP2.6 scenario. It should be noted that the drought indicator is defined as the change in the P5 multiplied by -1 . 


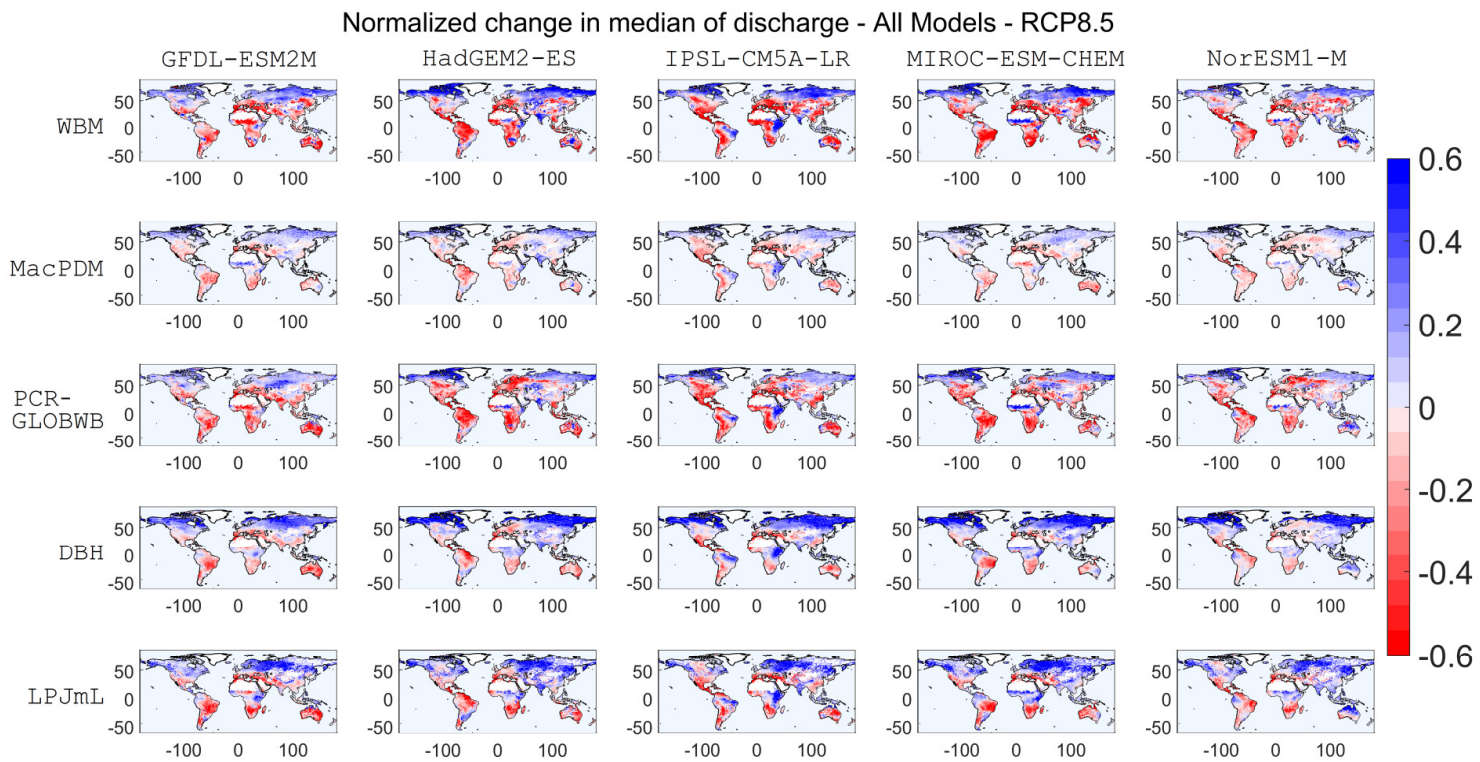

Figure S6. Normalized change in median of streamflow for each of GHM/GCM combinations under RCP8.5 scenario.

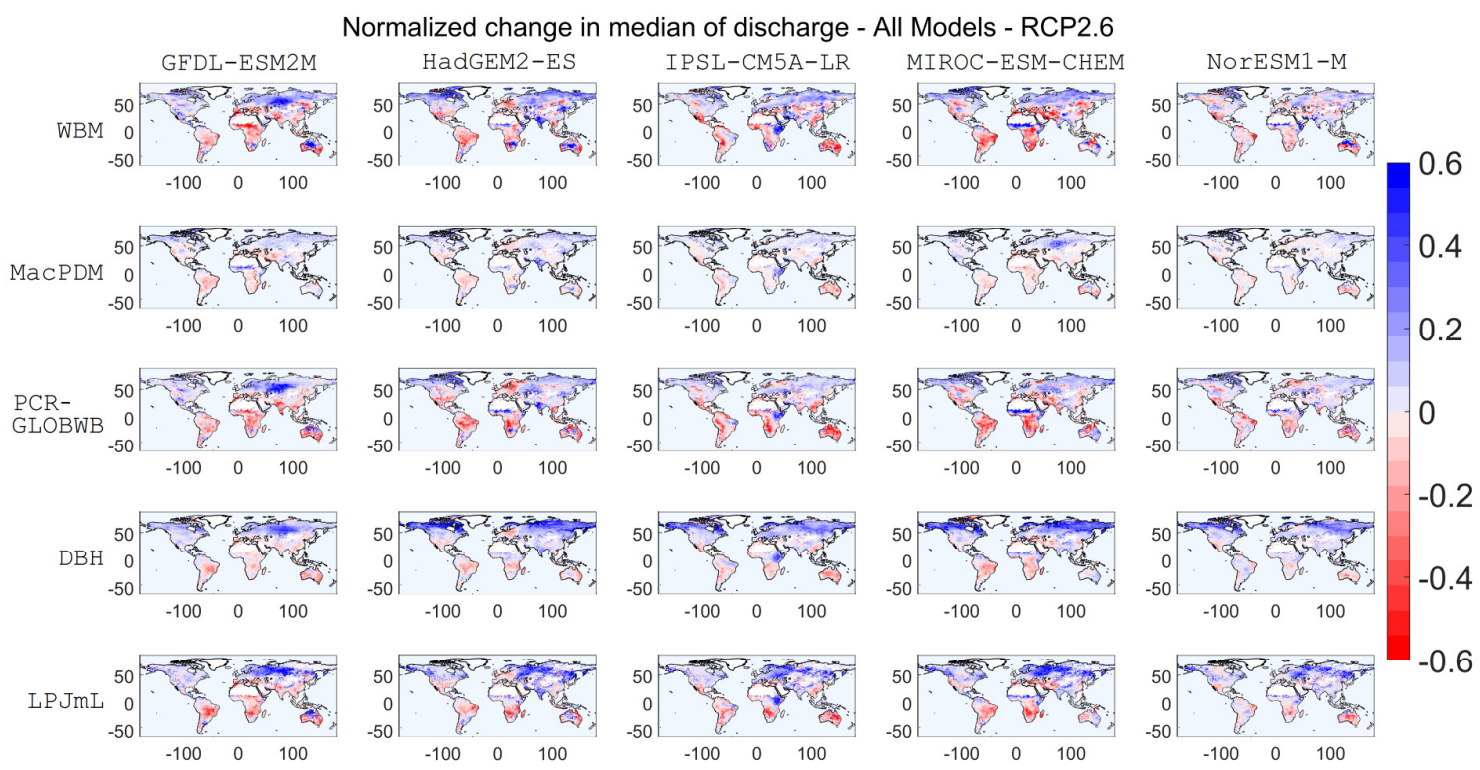

Figure S7. Normalized change in median of streamflow for each of GHM/GCM combinations under RCP2.6 scenario. 